\title{
Mapping EEG-Potentials on the Surface of the Brain: A Strategy for Uncovering Cortical Sources
}

\author{
Markus Junghöfer*, Thomas Elber+*, Paul Leiderer*, Patrick Berg*, and Brigitte Rockstroh*
}

\begin{abstract}
Sunmary: This paper describes a uniform method for calculating the interpolation of scalp EEG potential distribution, the current source density (CSD), the cortical porential distribution (cortical mapping) and the CSD of the cortical potential distribution. It will be shown that interpolation and deblurring methods such as CSD or cortical mapping are not independent of the inverse problem in potential theory. Not only the resolution but also the accuracy of these techniques, especially those of deblurring depend greatly on the spatial sampling rate (i.e., the number of electrodes). Using examples from simulated and real (64 channels) data it can be shown that the application of more than 100 EEC channels is not only favourable bul necesisary to guarantee a reasonable accuracy in the calculations of CSD or cortical mapping. Likewise, it can be shown that using more than 250 electrodes does not improve the resolution.
\end{abstract}

Key words: High sparial sampling EEG; Spatial deblurrirg; Current source density; Corrical Mapping; Sphenical spline interpolation lnverse problem.

\section{Introduction}

For any given distribution of the electrical potential on the surface of the head, there exists an infinite number of possible source configurations inside the head (Helmholiz 1885). This result from the laws of electrostatics presents a major problem when determining the source locations of MEG or EEG activity. A second theorem, however, maintains that the potential distribution on any closed surface which encloses all the generators can be determined uniquely from the surface potential map (Yamashita 1982; Conzalez et al. 1991). It is generally accepted that there are no sources between the scalp and the corlical surface. Therefore, the blurring of the electrical potential distribution which is caused by the high conductivity of the skull can in principle be compensated for by a mathematically unique transformation (Gevins et al. 1991, 1994). We refer to this transformation which then computes the potential distribution on an idealized and simplified cortical surface as "cortical mapping." It's uniquness does not depend on adequate modelling of conductivi-

- Department of Psychology and Department or Physics, University of Konstinz, Germany.

Accepted for publication lanuary 14,1997

This study was supported by grants from the Deutsche Forschungsfemeinschat to Thomas Ebert and Brigitte Rockstroh. The authors would like to thank Dr. Dorothy Charbonnier.

Correspondence and reprint requests should be addressed to Markus Junghöfer. Psychology Department. University of Konstanz. Unversitätsstraße 10, D-78434 Konstan2, Germany.

Copyright (c) 1997 Human Sciences Press, inc. ties or head shape. Inadequate modelling will "only" give rise to an inaccurate estimation of cortical surface potential. On the other hand this transformation is unique if, and only if, the electrical potential is known for the entire surface of the scalp. From a practical view we can only measure the potential at discrete locations (electrodes) and then estimate the potential at other locations. An alternative to cortical mapping which also compensates the spatial low pass that is characteristic for the transformation between cortex and scalp is current source density (CSD) calculation. In order to compute the CSD the potential on the scalp at sites between the electrodes must be determined by means of interpolation; the most common algorithm with which this is accomplished is the 4-nea rest-neighboursmethod. Spherical spline interpolation like the one suggested by Perrin et al. 1989 is, of course, more sophisticated.

This discussion leads us to two questions: 1) What are the consequences of the low pass characteristics of the skull, i.e., how strong is the spatial blurring and to what extent can it be compensated for by either cortical mapping or CSD analysis ? 2) Does the quality of either transformation depend on the number of electrodes?

In this paper we derive a procedure which allows for the computation of CSD and cortical mapping using a common algorithm. This approach emphasizes that spherical spline interpolation and with it the calculation of CSD on the one hand, and cortical mapping on the other hand, are equivalent procedures, and thus suffer from the same problems with respect to spatial sampling. 
We investigate these problems and illustrate the procedures using auditory evoked potentials recorded from 65 electrodes.

\section{Methods}

\section{Derivation of the procedure}

The CSD has to be derived from the interpolated scalp potential. A group of functions which automatically fulfills the physical constraint of minimal energy are Green's functions. In one dimensional space this leads to the cubic spline interpolation. However, all sets of functions within this space fulfill the required constraint of minimal energy (Freeden 1981; Wahba 1981). This complex situation may be illustrated for one dimension space by a shipbuilding analogy:

When building the body of a wooden ship, the wooden boards (spline) have to follow the skeleton of the ship body. A plank interpolates the space between points determined by the skeleton in a special way. It is a principle of nature, albeit a particular mathematical case in which the plank will bend itself so that the total energy of deformation is minimized. Considering the plank as a Green's function, it fulfills the constraint of minimal energy. The kind of wood, it's thickness, and it's elasticity can be chosen freely. Any type of plank would fulfill the requirements of minimum energy, but each type would interpolate the space in a different manner. A full set of plank types constitutes the space of Green's functions. Independent of the plank type, the interpolation will follow the desired form more closely if more positions are determined, i.e., the skeleton of the ship is denser. If we transfer this one dimensional case (without considering the width of the planks) into two dimensions, the space of the two dimensional Green's functions is obtained.

The potentials produced by electrically charged particles can equally be represented by means of Green's functions. For this reason it is possible to employ functions for the interpolation of scalp potentials that result from the charge distribution within the head. Thus, all possible charge distributions within the head span the space of Green's functions and in principle no single one is to be preferred above any other. Having the above example in mind, it becomes obvious that given the desired form of the body of the ship the thickness of the planks can also be determined. In the same way, it is possible when interpolating the EEG potential to choose a charge distribution that corresponds best to cortical activity.

\section{The Source Model}

The simplest case, a focal cortical activity, can be approximated by means of a stationary current dipole (Equivalent Current Dipole, ECD). Distributed sources can be described by a combination of such dipoles. Any scalp distribution can be accounted for if we assume one radially oriented dipole underneath each electrode. Having determined the strength of these dipoles the cortical surface potential can be computed by means of forward calculation. The optimal depth of these fictitious dipoles remains to be determined.

\section{Modelling the Volume Conductor}

In principal, a solution would be possible for any given arbitrary geometry and distribution of conductivity $\sigma$. Our model here assumes an isotropic volume conductor of spherical geometry composed of four homogeneous layers, each with a different conductivity (Cuffin and Cohen 1979). The layers model scalp, skull, cerebro-spinal fluid (CSF), and brain with

$$
\begin{gathered}
\sigma_{\text {brain }}=\sigma_{1}=\left(300 \Omega(\mathrm{cm})^{-1}, \sigma_{\mathrm{CSF}}=\sigma_{2}=3 \sigma_{\text {brain }}\right. \\
\sigma_{\text {skull }}=\sigma_{3}=\sigma_{\text {brain }} / 80, \sigma_{\text {scalp }}=\sigma_{4}=\sigma_{\text {brain }}
\end{gathered}
$$

The radius of the head $\mathrm{R}$ was assumed to be $9.20 \mathrm{~cm}$, the radius of the brain $8.15 \mathrm{~cm}$, the thickness of skull and scalp were each set to $4.2 \mathrm{~mm}$, and the thickness of the CSF to $2.1 \mathrm{~mm}$.

Of course, the practical determination is limited by the quality of measurement as well as by the adequacy of the model, i.e., geometry and conductivities. Yamashita and Takahashi (1984) compared the actual values of epicardial potentials with those determined from torsomeasured potentials, and showed that a good agreement can be achieved if the signal-to noise-ratio becomes sufficiently large. Similar results should be expected for the head since both systems resemble each other in terms of the physical equations.

\section{Choice of Green's spherical spline function}

As indicated above, we will illustrate the procedure for radial sources. The generalisation to arbitrarily oriented current dipoles is straightforward. The expression in spherical coordinates for the potential produced by one single, radially directed current dipole $\stackrel{\perp}{p}$ on the surface of the sphere is given by (Cuffin and Cohen 1979):

$$
V(\phi, \theta)=p \cdot \sum_{n=1}^{\infty} P_{n}^{0}(\cos \theta) \cdot \Gamma^{s \operatorname{col} p}(n)
$$

$$
\Gamma^{\text {salp }}(n)=\frac{1}{4 \pi \sigma_{1} R^{2}} \cdot \frac{(2 n+1)^{4} \cdot f^{n-1} \cdot(c d)^{2 n+1}}{\Gamma(n)}
$$




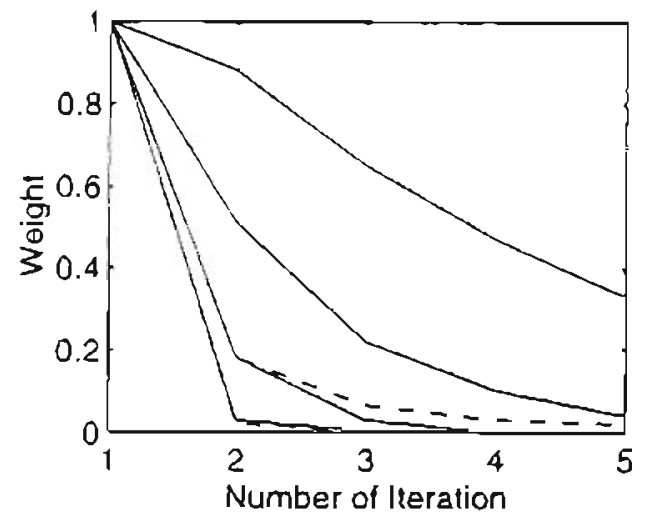

Figure 1. Solio lines; weight function of the Legendre polynomials in equation 2 of different depths: $10.5,39.5$. 66.5 and $79 \mathrm{~mm}$ (from top to bottom) under the cortical surface or respectively $71,42,15$ and $2.5 \mathrm{~mm}$ measured from the centre of sphere. Dashed lines: weight function of equation 9 with $m=2$ (top) and $m=4$ (bottom).

$$
\begin{aligned}
& \Gamma(n)=d^{2 n+1} \cdot\left[\begin{array}{c}
b^{2 n+1} n\left(\frac{\sigma_{1}}{\sigma_{2}}-1\right)\left(\frac{\sigma_{2}}{\sigma_{3}}-1\right)(n+1)+ \\
c^{2 n+1}\left(\frac{\sigma_{1}}{\sigma_{2}} n+n+1\right)\left(\frac{\sigma_{2}}{\sigma_{3}} n+n+1\right)
\end{array}\right] . \\
& {\left[\left(\frac{\sigma_{3}}{\sigma_{3}} n+n+1\right)+(n+1)\left(\frac{\sigma_{3}}{\sigma_{1}}-1\right) d^{2 n+1}\right]+} \\
& (n+1)+c^{2 n+1}\left[\begin{array}{c}
b^{2 n+1} n\left(\frac{\sigma_{1}}{\sigma_{2}}-1\right)\left(\frac{\sigma_{2}}{\sigma_{3}} n+\frac{\sigma_{2}}{\sigma_{3}}+n\right)+ \\
\left.c^{2 n-1}\left(\frac{\sigma_{1}}{\sigma_{2}} n+n+1\right)\left(\frac{\sigma_{2}}{\sigma_{3}}-1\right)\right] \\
{\left[n\left(\frac{\sigma_{2}}{\sigma_{1}}-1\right)+\left(\frac{\sigma_{3}}{\sigma_{1}} n+\frac{\sigma_{3}}{\sigma_{1}}+n\right) d^{2 n+1}\right]}
\end{array}\right]
\end{aligned}
$$

Whereby,

$\phi=$ azimutal and $\theta=$ polar angle of spherical head model

$R=9.2 \mathrm{~cm}$, radius of the head

$f=g / R$ excentricity of the dipole locations

$g=$ radius of the fictitious dipoles in $\mathrm{cm}$ (this is the only parameter to choose in the radial case)

$b, c$ and $d$ describe the relations between the radii of the shells:

$b=8.15 / R ; c=8.36 / R ; d=8.78 / R$

$P_{r}=P_{n}^{0}$. Legendre Polynomiais or zonal spherical surface functions.

The potential $V(\phi, \theta)$ is only dependent on the angle between the vectors describing the site of measurement and the dipole location. Given N electrodes, there will be $N$ angles $\alpha i j$ for each particular electrode j, and the potential will be determined by the corresponding sum across the fictive dipoles under all electrode sites:

$$
V_{j}(t)=\sum_{i=1}^{N} p_{i}(t) \sum_{n=1}^{\infty} p_{n}\left(\cos \alpha_{i j}\right) \cdot \Gamma^{\text {scalp }}(n)
$$

In matrix notation the solution will be an eigenvalue problem:

$$
\begin{gathered}
\underline{V}=\underline{\underline{p}} \underline{\underline{G_{\text {imn }}^{\text {sealp }}}} \\
\underline{\underline{G_{i n D}^{\text {scolp }}}}=G_{i j}^{\text {scalp }}=\sum_{n=1}^{\infty} P_{n}\left(\cos \alpha_{i j}\right) \cdot \Gamma^{s c a l p}(n)
\end{gathered}
$$

being an $\mathrm{N} x \mathrm{~N}$ symmetrical matrix which depends solely on the model parameters and on the geometrical configuration of the electrode asray. For any given electrode array, $\underline{G_{\text {ino }}^{\text {scalp }}}$ can be computed first, and then, given a measurement of $\underline{V}$ the solution for the vector of dipoles $\underline{p}$ can be calculated. If the dipole vector $\underline{p}$ has been determined, the potential distribution on the surface of the head, which so far was only known for the electrode position (forward solution), can now be calculated for any number of points $k_{\text {scalp }}=\left[k_{l_{\text {kalp }}}, \ldots, k_{M_{\text {scalp }}}\right]$ :

$$
V_{k_{\text {sout }}}(t)=\sum_{i=1}^{N} p_{i}(t) \sum_{n=1}^{\infty} P_{n}\left(\cos \alpha_{i k_{n=1 / p}}\right) \cdot \Gamma^{s c a l p}(n)
$$

If one compares this formula with that of Perrin et aL 1979:

$$
\begin{gathered}
V_{k_{\text {sat }}, t}(t)=\sum_{i=1}^{N} p_{i}(t) \sum_{n=1}^{\infty} P_{n}\left(\cos \alpha_{i k_{\text {xntr }}}\right) \cdot \Gamma^{P e r i n}(n) \\
\Gamma^{\text {Perin }}(n)=\frac{2 n+1}{n^{m}(n+1)^{m}}
\end{gathered}
$$

with m 22 a constant to be chosen arbitrarily, the similarities between the two procedures become evident. If one compares the normalised course of realp for different excentricities $\mathrm{f}$ (different radii $\mathrm{g}$ of fictitious dipoles) with $\Gamma^{P t r i n}$ for different values of $m$ (figure 1), it becomes obvious that equation 8 corresponds to equation 7 when choosing corresponding f values. Generally, $\Gamma^{\rho}$ errin can be considered a special case of the more general approach realp ( $n$ ) (In addition to a free choice of depth, as in the latter case, thicknesses and conductivities of the different layers can also be freely chosen).

In matrix notation equation 7 reads : 


$$
\underline{\underline{V}}=\underline{\underline{p}} \underline{\underline{G_{f o r}}}
$$

In this case:

$$
\underline{\underline{G_{i o r}^{i c a l p}}}=G_{i k}^{i c a l n}=\sum_{n=1}^{\infty} P_{n}\left(\cos \alpha_{i k}\right) \cdot \Gamma^{i \operatorname{colp}}(n)
$$

is generally an asymmetric $\mathrm{N} \times \mathrm{M}$ matrix which depends solely on the model parameters, on the geometrical configuration of the electrode array, and on the desired scalp locations.

The eigenfunctions of the Operator in spherical coordinates are the spherical surface functions $Y_{l}^{m}$ with $-1(l+1)$ as its eigenvalues:

$$
\Delta_{\otimes, \varphi} Y_{l}^{\prime \prime}(\vartheta, \varphi)=-l(l+1) Y_{l}^{n !}(\vartheta, \varphi)
$$

Thus, the two dimensional spherical Laplacian of a Legendre polynomial equals a multiple integer of the same function,

$$
\Delta P_{n}=-n(n+1) P_{n}
$$

and we can now caiculate the current source density of the interpolated potential distribution on the scalp in a very simple manner:

$$
\begin{aligned}
& \underline{\underline{C^{\text {SCalp }}}}=\underline{\underline{p}} \underline{\underline{G_{j o r}^{C S D \text { Colp }}}} \\
& \underline{\underline{G_{\text {for }}^{\text {CSOscaip }}}}=G_{i \mathrm{isel}_{\text {ser }}}^{\text {CSD:culy }}= \\
& -\sum_{n=1}^{\infty} P_{n}\left(\cos \alpha_{i k_{\text {cail }}}\right) \cdot n \cdot(n+1) \cdot \Gamma^{\text {couly }}(n)
\end{aligned}
$$

Up to this point the present procedure served to interpolate the scalp potential and to use the interpolated scalp potential to compute the CSD. An interesting property of the procedure is that the calculated dipole moments $\underline{p}$ can also be used to estimate the potential distribution on shell covering the cortical surface (cortical map).

Let $k_{c 0 r t}=\left[k_{1_{1}: m}, \ldots, k_{M_{\text {cort }}}\right]$ denote any number of points on a shell enclosing the cortical surface

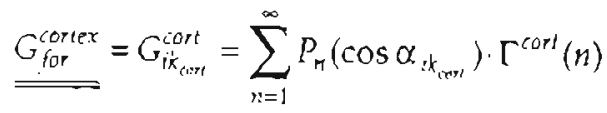

whereby $G_{\text {for }}^{\text {cortex }}$ is again a matrix which can be determined independently of a particular measurement.

$$
\Gamma^{\text {corlex }}(n)=\left[\frac{\Omega(n) \cdot(n+1)}{\Gamma(n) \cdot R}+n\right] \cdot\left(\frac{f}{b}\right)^{n} \cdot \frac{1}{b \cdot f} \cdot \frac{1}{4 \pi \sigma_{1} R^{2}}(17)
$$

with $b$, the assumed relative radius of the brain (cortical sphere), and

$$
\begin{aligned}
& \Omega(n)=\left[d^{2 n+1} n\left(\frac{\sigma_{3}}{\sigma_{4}} n+n+1\right)+d^{4 n+2} n(n+1)\left(\frac{\sigma_{3}}{\sigma_{4}}-1\right)\right] \\
& {\left[c^{2 n+1}\left(\frac{\sigma_{1}}{\sigma_{2}}-1\right)\left(\frac{\sigma_{2}}{\sigma_{3}} n+n+1\right)+b^{2 n+1}\left(\frac{\sigma_{1}}{\sigma_{2}} n+\frac{\sigma_{1}}{\sigma_{2}}+n\right)\left(\frac{\sigma_{2}}{\sigma_{3}}-1\right)\right]} \\
& +\left[\begin{array}{c}
c^{4 n+2} n(n+1)\left(\frac{\sigma_{2}}{\sigma_{2}}-1\right)\left(\frac{\sigma_{2}}{\sigma_{3}}-1\right) \\
\quad+b^{2 n+1} c^{2 n+1}\left(\frac{\sigma_{1}}{\sigma_{2}} n+\frac{\sigma_{1}}{\sigma_{2}}+n\right)\left(\frac{\sigma_{2}}{\sigma_{3}} n+\frac{\sigma_{2}}{\sigma_{3}}+n\right)
\end{array}\right] \\
& \cdot\left[c^{2 n+1} n\left(\frac{\sigma_{3}}{\sigma_{4}}-1\right)+c^{2 n+1} d^{2 n+1}\left(\frac{\sigma_{3}}{\sigma_{3}} n+\frac{\sigma_{3}}{\sigma_{4}}+n\right)\right]
\end{aligned}
$$

Again we can determine the current source density of the cortical potential distribution as:

$$
\begin{aligned}
& \underline{C^{c o r t}}=\underline{p \cdot G_{f o r}^{C S D c o r t}}
\end{aligned}
$$

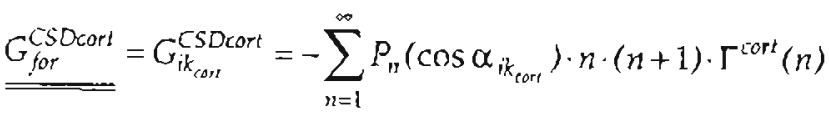

As it has been mentioned, these formulae rely on fictitious radial sources with exactly defined locations. It is possible to generalise these formulae to arbitrarily oriented and arbitrarily located dipoles. However, this does not solve the basic problem that results from the lack of knowledge of the source parameters. In order to compensate for this missing information, one of the following three procedures may be applied: [1] The assumed radially oriented dipoles are located deep inside the head, i.e., a small eccentricity $f$ value is chosen so that the contribution of higher Legendre polynomials become reduced, and hence the interpolated distribution becomes as smooth as possible. The lower limit off constitute the numeric singularity of the calculations. [2] The depth of the fictitious dipoles can be chosen so that the error estimated for all kikely or mearingful source configurations will be minimised. [3] Increasing the spatial sampling rate (number of electrodes) is a third obvious but not necessarily practical possibility.

In order to facilitate the choice between these procedures, we may again go back to the shipbuilding analogy 

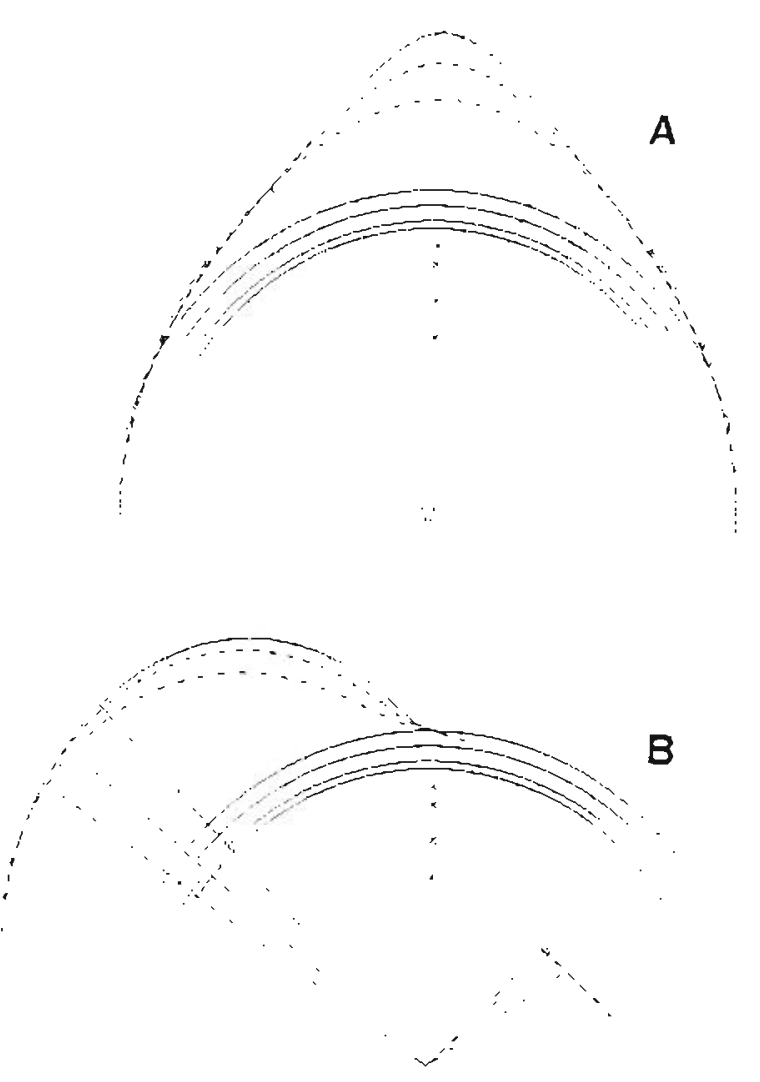

Figure 2. Potential and wioths of four different radial A) and tangential $B$ ) dipoles in the depths of $5,10,20$ and 30 $\mathrm{mm}$. Dipole locations are marked with $x$. The amplitudes are given by the rodial distances between scolp and potential. Potentials are positive above and negative below scalp.

for guidance. Assume that the skeleton of the ship is given, but that the desired form of the ship is unknown. If a ship builder were to proceed according to [1], he or she would use as limited as possible flexible planks, based on the assumption that the curvature between two points on the skeleton is minimal. In cases where no additional information is available, as in Perrin et al. 1987,1989, this procedure must be applied. The lower limit of the numerical singularity can hereby be made clear also. The splines may of course be so stiff that they can be adjusted to the skeleton using a reasonable force. This depends on its shape. The necessary accuracy of calculation corresponds to the expanded force. Using the international 10/20 system Perrin spline functions with a $m=4$ could be chosen. In a 65 channel design the matrix which has to be inverted becomes extremely singular. When applying 128 electrodes the accuracy is not sufficiant. In this case more flexible splines with greater proportion of higher spatial frequencies have to be used for instance by using $m=3$ or $m=2$.

In the second case, the additional information i.e., the desired curvature of the planks, is available to the ship- builder, and therefore the type of planks can be chosen which results in the smallest error. However, this procedure requires complex computations.

The third approach, i.e., the increment of points (electrodes) is the ondy procedure which ensures that no errors are made when determining the form.

The choice of an adequate spline function depends to a large degree on the particular spatial sampling. Using the procedure introduced in this paper, it would require optimising the choice of eccentricity f of the dipole location. A simpler possibility would be to choose the optimal eccentricity and then restrict the number of polynomials that are used in the interpolation depending on the spatial sampling. With the transition from the space of Green's function to the interpolation with polynomials the Nyquist (sampling) theorem has to be taken into account defining an upper limit for the polynomials to be used. In the following section using noise-free, synthetic data, we will show how well the described procedures are able to interpolate the predetermined potential distributions and which errors might occur. The calculations from the examplary data set will also show the extent to which cortical sources become blurred on the scalp and the extent to which CSD and cortical mapping may assist in debluming depending on the electrode density.

\section{Results}

\section{Simulated Sources - the problem of forward solution}

The dependency of potential blurring on the orientation and depth of the dipole can be inferred from figure 2. It illustrates the potential distribution of radial and tangential sources at four different depths $(5,10,20$ and $30 \mathrm{~mm}$ below the cortex). Even the scalp distribution of shallow sources, i.e., those close to the scalp, are considerably blurred. The half width (the diameter of the circle where the potential is greater than half the maximum) of the distribution can be used as a measure for blurring in the case of radially oriented dipoles. For tangentially oriented dipoles it is more illustrative to use the distance between maximum and minimum of the corresponding lead field. The half widths are also seen in figure $2 a$ and 2b. The dependency of half widths on eccentricity is ilustrated in more detail in figure 3 (lower part, solid line). It is clear that in the chosen volume conductor, the half width increases nearly linearly with the depth of the dipole location.

The potential and CSD distribution that result from the three different deblurring methods are illustrated in figure 4 for a radial and a tangential dipole located at a depth of $10 \mathrm{~mm}$ (radius $=71.5 \mathrm{~mm}$ ). Both CSD and cortical mapping increase the spatial resolution considerably and 

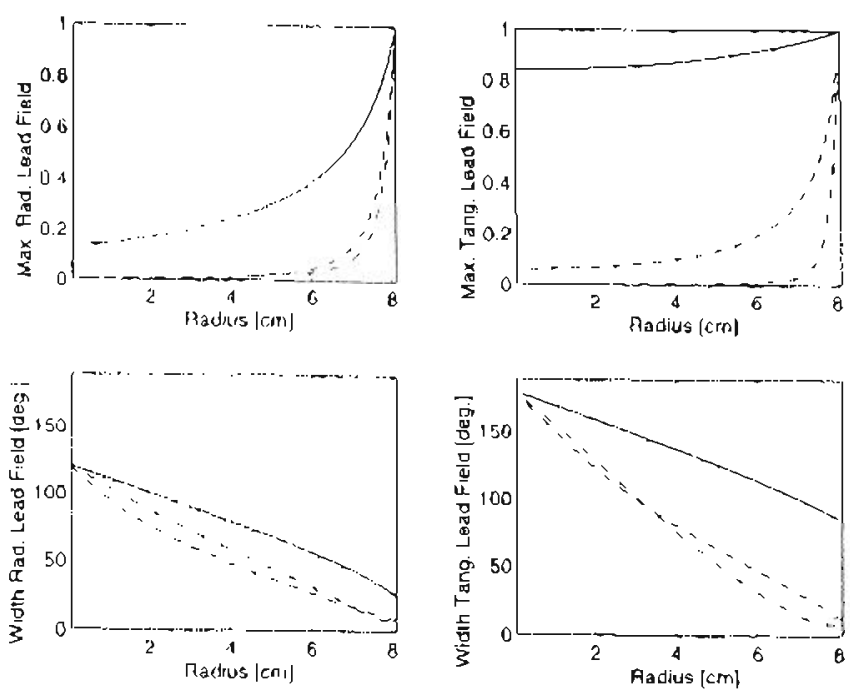

Figure 3. Top: Maximum normalized leadfieid amplitudes of radial (left) and tangential (right) dipoles as a function of eccentricity. Bottom: half-wiaths as a function of eccentricity. solia line: Scalp-lead field; dashed line: ScalpCSD-lead field; dashed-dotted Cortical-lead field; dotted line: Cortical-CSD-lead field.

\section{A}

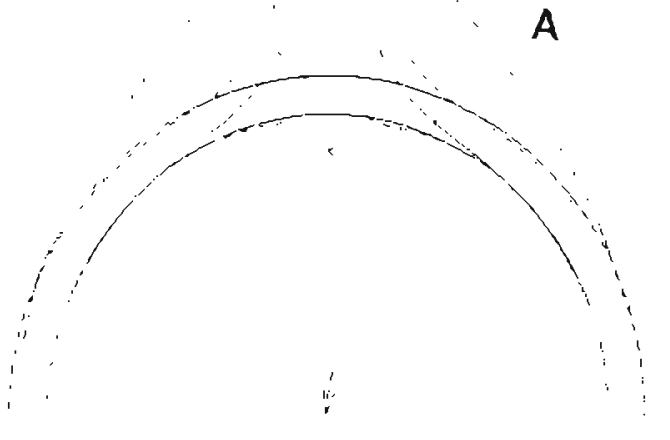

Figure 4. Scalp-potential (upper solid line), scalp-CSD (upper dashed line), cortical-potential (lower solld line) and cortical-CSD (lower dasheo line) of A) a radial source at depth of $10 \mathrm{~mm}$ and 8 ) a tongential dipole of depth of 20 $\mathrm{mm}$. both procedures result in the same half width. However, using the CSD of the cortical map, the half width can be reduced even further. The remaining lines in figure 3 illustrate this effect. Normalised leadfields (top) and half widths (bottom) for CSD (dashed line), Cortical Potential (dashed-dotted line) and the CSD of Cortical Potential (dotted line) are shown as a function of eccentricity.

The value of these procedures in determining source locations becomes apparent when four simple configurations of two radial dipoles are compared (see figure 5). Even if the distance between the sources is large, at 45 degrees, and even if the location is shallow at a depth of $10 \mathrm{~mm}$, the scalp potential distribution itself hardly discriminates the presence of two separate sources (figure $5 a$ ). With increasing depths (figure $5 b$ ), and for smaller distances between the sources (figure 5c) the situation worsens. For deeper closely spaced sources, only the calculation of the cortical CSD results in a satisfying resolution of the source space (figure $5 d$ ). Results for the tangentially oriented sources are qualitatively the same.

So far, the results were obtained by solving the forward problem using a sampling rate of 256 points (electrodes) on the sphere. The resolution obtained carnot be transferred to the inverse problem, when source locations are usually not known and the sampling rates are significantly smaller.

\section{Simulated sources - the inverse problem}

The extent to which the quality of interpolation and
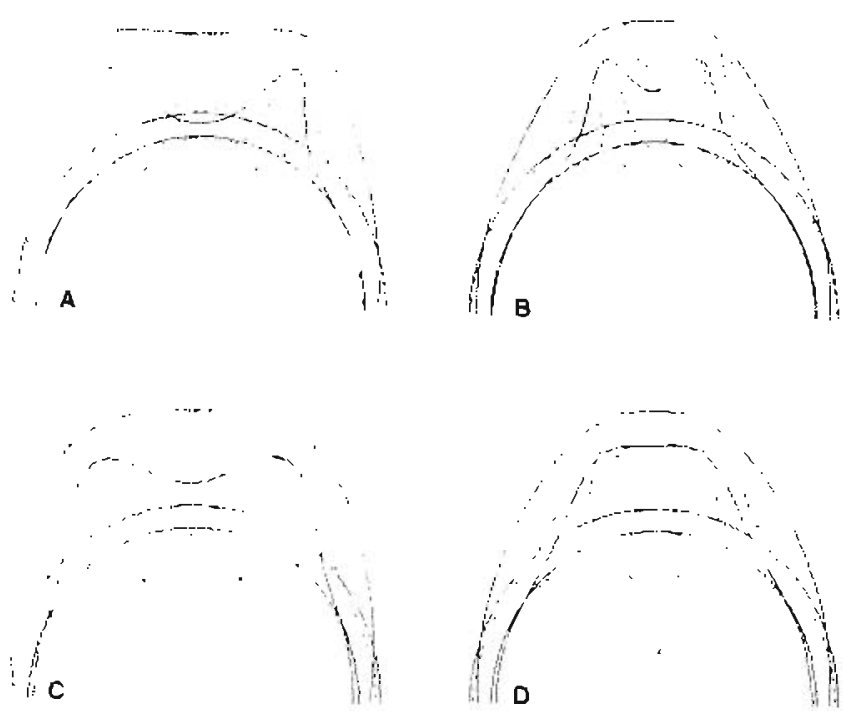

Figure 5, scalp-potential (upper solid line), scalp-CSD (upper dashed line), cortical-potential (lower solid line), and cortical-CSD (lower dashed line) due to two radial dipoles at a depth of $10 \mathrm{~mm}$ separated by A) 45, 8) 22.5 degrees, and of a depth of $20 \mathrm{~mm}$ sperated by C) 45 , and D) 22.5 degrees. Locations are marked with $x$. 

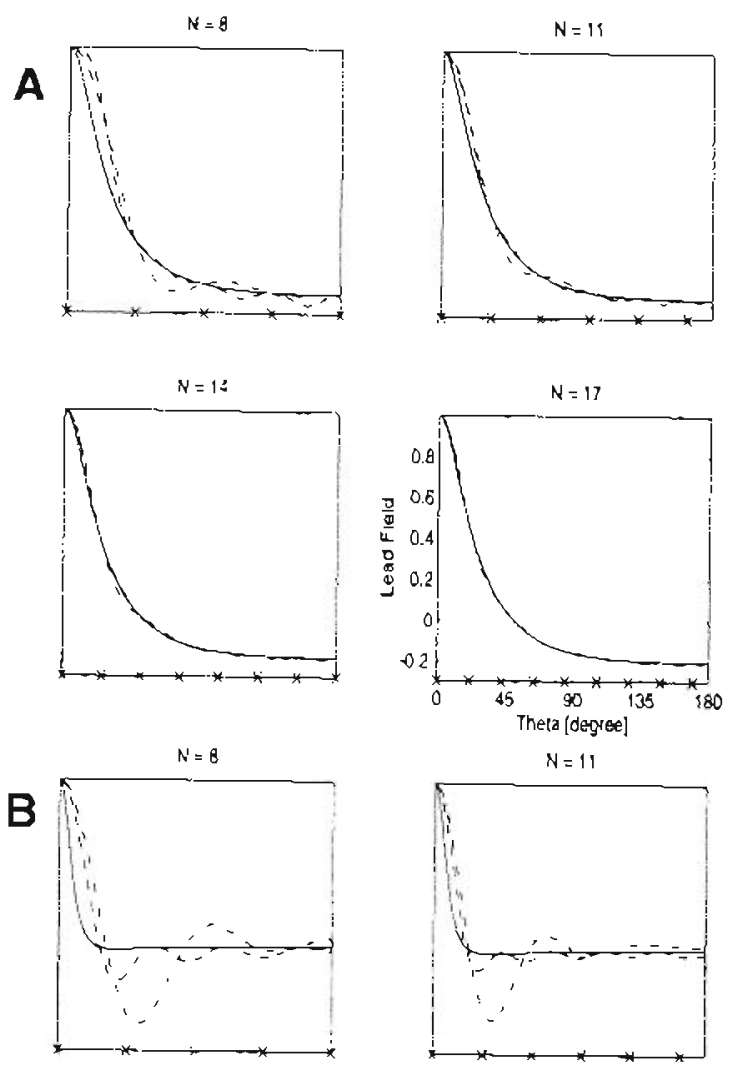

$N=11$
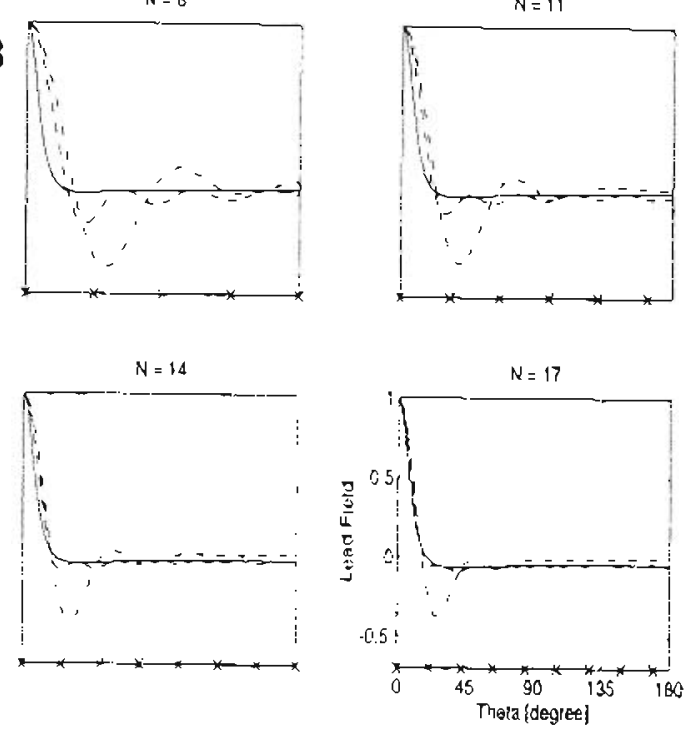

Figure 6. Relationship between goodness of interpolation and electrode spacing: A) Scalp-potential (solid line) due to a radial dipole at a depth of $10 \mathrm{~mm}$. B) CSO (solid line) of the same potential. Interpolation of this potentlal / CSD with a Perrin algorithm (dash dotted) and the optimum spline algorithm (dashed) with different electrode spacings. $8,11,14$ and 17 electrodes on a circle correspond to approximately $25,50,75$, and 120 electrodes on the head.

deblurring methods depends on the sampling rate becomes obvious from the calculations illustrated in figure 6. The dependency of the quality of the interpolation and deblurring methods on the sampling rate is highlighted in figures 6 and 7 . In these cases, the potential due to a radially oriented dipole, situated at a depth of $10 \mathrm{~mm}$ below the scalp, has been predetermined at only a few electrode sites. These locations are indicated by an ' $x$ ' on the abscissa in figures 6 and 7 . Based on these discrete
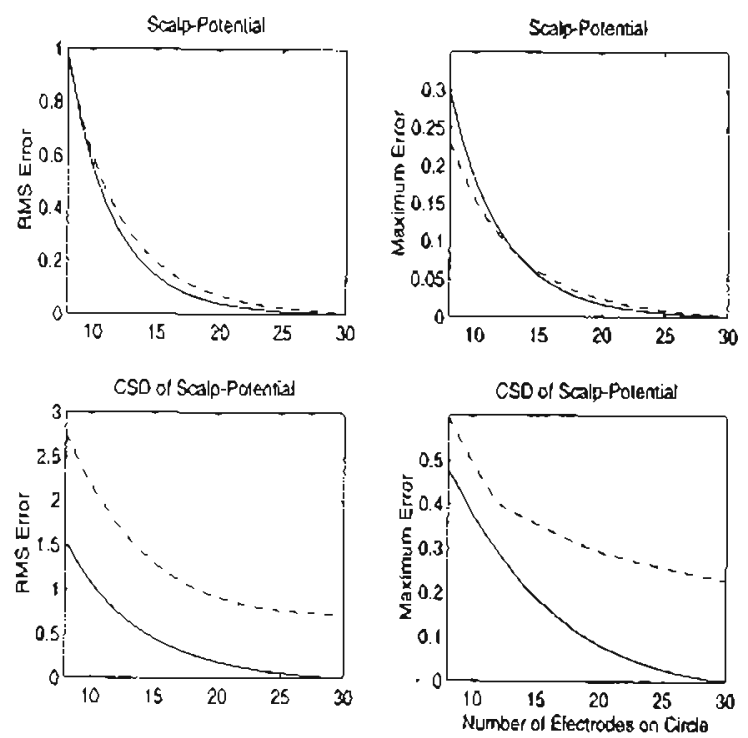

Figure 7. RMS enor (lett) and maximum error (right) of the scalp-potential interpolations (top) and the CSD calculafions (bottom) in dependency of sampling rate as shown in figure 6 . The solid line corresponos to the errors of the optimal algorithm while the dashed line shows the errors of the Perrin algorithm.

potential data, the procedures described in the previous sections served to reconstruct the (in this case known) potential distribution and CSD. The resulting maximum deviation and the RMS 'error' are shown in figure 7 . We put 'error' in quotation marks to remind the reader that there are an infinite number of different potential distributions that fit exactly to the potential at the selected points. Irrespective of this 'non-uniqueness', figure 7 shows that the maximum and the RMS errors are clearly reduced even beyond electrode numbers providing a resolution of $18 / 2 \pi$. This corresponds (see figure 9) to a 130 channel EEG-system. For illustrative purposes ondy, Perrin's interpolation has been selected in figures 6 and 7 . Even at high spatial sampling the calculation of the CSD in particular may result in considerable errors if sources are located close to the surface. This is due to the insufficient contribution of higher Legendre components (see figure 1). The spline functions are too 'stiff in order to adequately approximate the steep potential distribution of shallow radially oriented sources. As can be derived from figure 4 (lower left) this problem varishes with increasing depths of the source locations. The same is true for tangentially oriented sources which produce smaller amplitudes of higher spatial frequency than radial sources at the same depth (figure 4 (lower right)). It is not appropriate to compare Perrins's interpolation with the one resulting when the source configuration is exactly known.

Apart from the error estimation, calculations of the corresponding half widths (figure 8) also show a drastic 

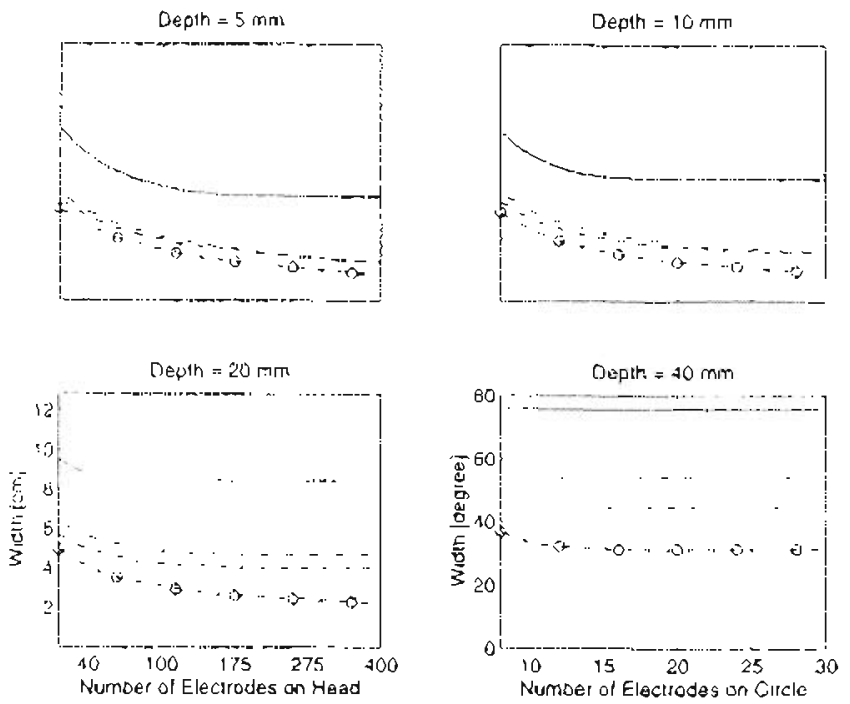

Figure 8. Changes in half-width of scalp-potential (solid line), CSD (dashed line), cortical surface distribution (aashed dotted line) and CSD of cortical surface distribufion (Cashed circled line) in dependency of spotial sampling frequency (i,e, number of electrodes). Right axis: number of electrodes on a circle $(2 \pi)$ of the spherical head. Left axis (corresponding to the right axis): number of electrodes on the whole heod excluding the dorsal neck region. A head region of 225 degrees is assumed. Right ordinate: half-width in degree: left ordinate (corresponding to the right axis): holf-width in $\mathrm{cm}$. Calculations were bosed on a one-dimensional spherical coordinate system ( $r$, phi=const) and a radial dipole at a depth of $5 \mathrm{~mm}, 10 \mathrm{~mm}, 20 \mathrm{~mm}$, and $40 \mathrm{~mm}$. Optimal spline functions were used

dependency of the resolution on the spatial sampling. Even without employing the deblurring methods, considerable achievements can be reached for shallow sources up to an electrode spacing of a $16 / 2 \pi$ using simple interpolation (upper figure 8 ). Deeper sources produce smaller power at higher spatial frequencies, and thus, the erhancement of the spatial resolution is less dependent on electrode spacing (lower figure 8). From figure 8 (lower right), for instance, it may be concluded that independent of spatial sampling it is impossible to resolve two radial sources at a depth of $4 \mathrm{~cm}$ seperated by less than approximately 35 degrees. If the CSD is calculated or the cortical map or even the CSD based on the cortical map, this dependency becomes more excessive. In this case a high number of electrodes (256) can be useful. Considering a radially oriented source at a depth of $10 \mathrm{~mm}$ (i.e., a source located in a cortical gyrus), the half width of the scalp distribution would amount to as much as 55 degrees when the standard $10 / 20$ system is used. This number would drop to about 15 degrees for a 128 channel system. The resolution enhancement

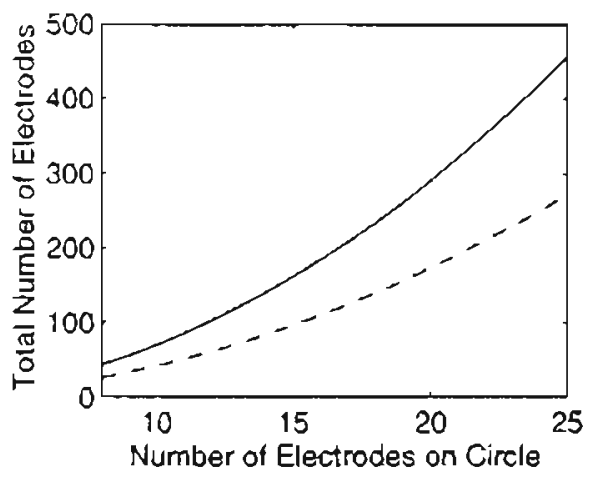

Figure 9. Relationship between number of electrodes on a circle $k$ and the number of electrodes on a sphere $E$ or on the realistic head $G$ with generallsed number of electrodes with 225 degree between nasion and inion. In the 10.20 system the electrodes LPA, C3, Cz, C4, and RPA constitute a semi-circle. Adding the posittons opposite to C3. $\mathrm{C} z$ and $\mathrm{C} 4$ results in 8 electrodes $/ 2 \pi$.

would then amount to more than $350 \%$.

The combined inspection of figures 7 and 8 allows the following early conclusion: The application of deblurring methods allow for considerable improvement in the fidelity and spatial resolution of potential distributions derived from EEG-recordings. The deblurring, however, must be based on actual measurements with sufficient spatial resolution; Interpolations are not sufficient to produce correct results. Figure 9 demonstrates the relationship between number of electrodes on a circle $k$ and the number of electrodes on a sphere $E$ or on the realistic head $G$ with generalised number of electrodes with 225 degree between nasion and inion. Here we used the following calculation: $\quad G=\left(1-\frac{\left(h^{2}+2 h\right\rangle}{4}\right) \cdot E$; $E=\frac{4 \pi}{3 \cdot a \cos \left(\frac{\cos (\gamma) \cdot(1-\cos (\gamma))}{\sin ^{2}(\gamma)}\right)-\pi} ; h=1-\cos \left(\frac{67.5 \pi}{180}\right) ;$ $\gamma=\frac{2 \pi}{k}$

\section{Choice of a useful spline function}

So far, we have assumed that the location and the orientation of the sources are known. In this section, we want to demonstrate the inaccuracies that arise from erroneous interpolations which are based on a wrong choice of the splines, i.e., erroneous assumptions of the location and/or the orientation of the dipolar source.

Consider a radial source, located at a distance of $r=40$ $\mathrm{mm}$ from the centre of the head. Corresponding to the standard $10 / 20$ locations we assume that the potential is 

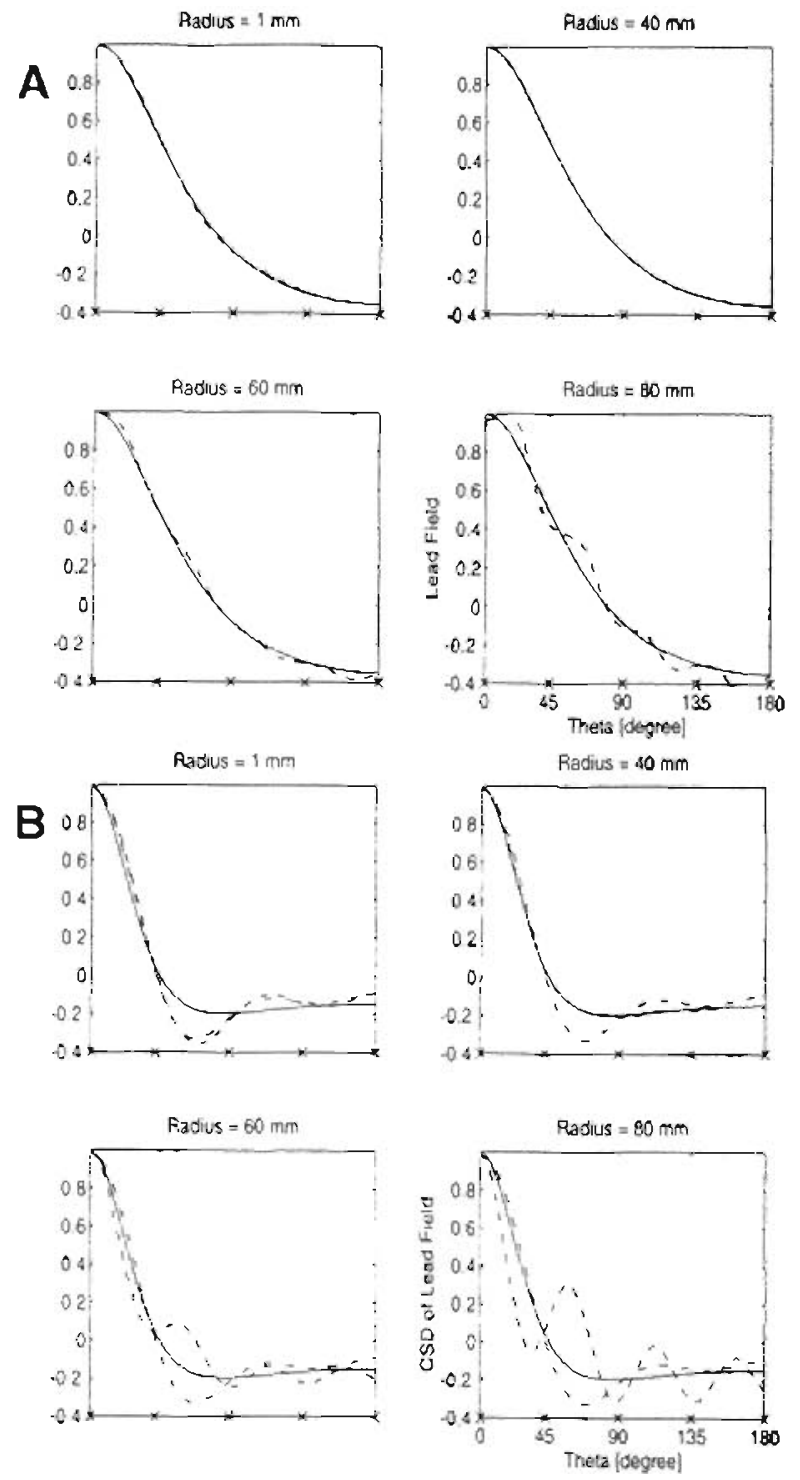

Figure 10. (A) Relationship between the qually of interpolation and spline-dipole-radius with radii of 1,40,60, and $80 \mathrm{~mm}$. Solid line: Right branch of the lead-field due to a radial dipole at a depth of $41.5 \mathrm{~mm}$ (radius $=40 \mathrm{~mm}$ ). Dashed line: Interpolation on the basis of $\overline{8} / 2 x$ electrodes (marked with $x$ ). The oplimally selected splines $(r=40 \mathrm{~mm})$ produce the best adoptation. B) The CSD of the lead - field. Doshed dotted line: CSO using the weighting function, $(2 n+1) /\left(n^{m-1}(n+1)^{m-1}\right), m=4$ (Perrin).

measured at eight equidistant locations around the circle and then interpolated using radial spline functions of varying depths. Figure 10 compares the calculations for the interpolated values with an optimal choice of the spline function (radius $=40 \mathrm{~mm}$ ) and an unfavourable choice. The solid line always refers to the 'true' symthetic scalp-potential of the radial source at $r=40 \mathrm{~mm}$. The dotted line corresponds to the interpolation with radii of
A

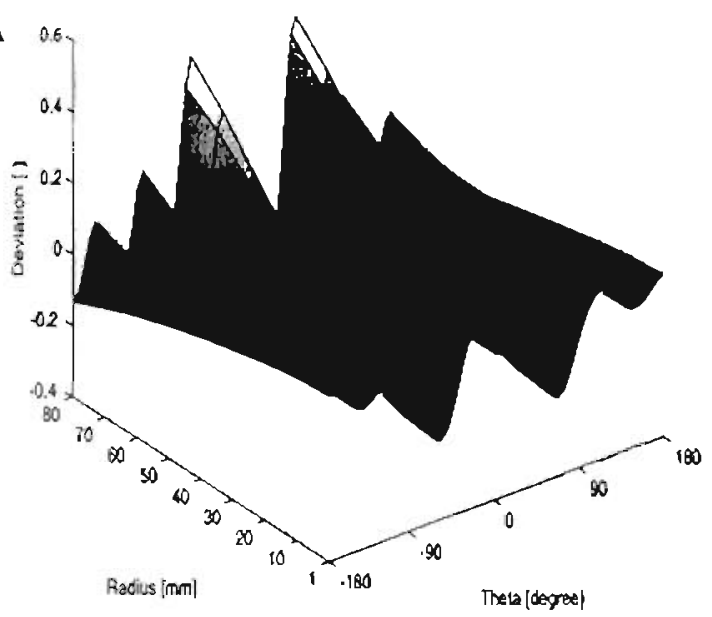

B

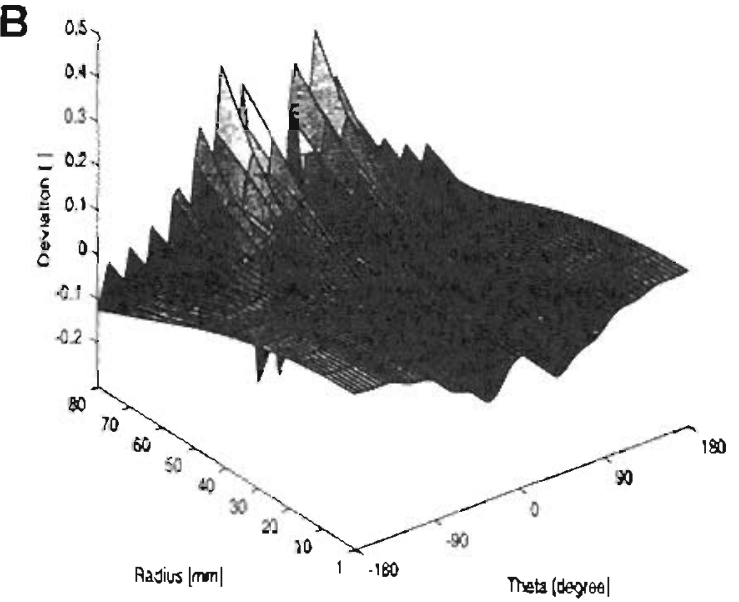

Figure 11. Deviation between CSD due to lead-field dipole (described in figure $10 \mathrm{~b}$ ) and calculated (interpolated) CSO of Leadfield (figure 1Ob) with different spline-dipole-radii and a sampling rate of a) $8 / 2 \pi$, and $b$ ) $16 / 2 \pi$.

$1 \mathrm{~mm}$ (i.e., the source was assumed to be deeper) and 60 and $80 \mathrm{~mm}$ (a shallower source). The errors become particularly large when the spline-dipole-radii have been chosen too large, i.e., the spline functions ('planks') are too 'soft'. When the CSD is computed, (figure 10b) it becomes obvious that choosing too 'stiff spline functions (spline-dipole at a too great depth) will also not fit the lead field optimally. The dashed-dotted line corresponds to the calculation using the spline suggested by Perrin et al. $(m=4)$, a choice that implies a very 'stiff (see figure 1) spline function.

This error in choice is evident from the comparisons in figure 11 and 12. Figure 11 shows the deviations from the synthetic CSD for the CSD calculated from interpolations based on nine different dipole-radii. Figure 12 illus- 

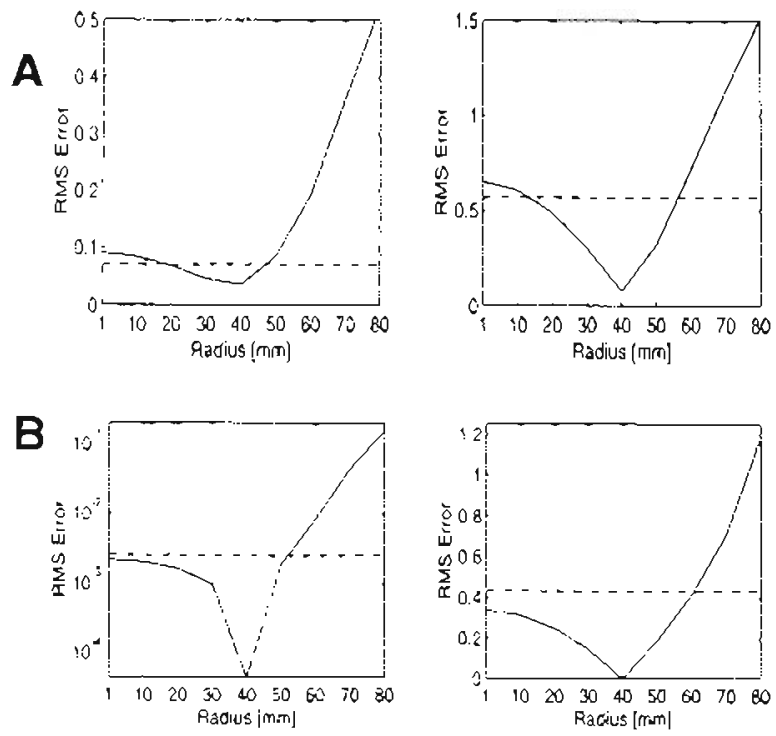

Figure 12. Solid line: Root mean square (RMS) of the deviation of lead-field (shown in figure 10) and CSD of lead-field (shown in figure 10 and figure 11) with o sam. pling rate of $8 / 2 \pi$ (upper left and right), and $16 / 2 \pi$ (lower left and right). Dashed line: using the Perrin weighting function with $m=4$.

trates the respective RMS errors.

For the sake of clarity, the results employed a low spatial sampling $(\delta / 2 \pi)$. When a denser sampling is used, results remain qualitatively equivalent (figure $11 \mathrm{~b}$ and 12b). An example is shown in figure 13, a radially oriented source was assumed at a depth of $20 \mathrm{~mm}$ and a theta-angle of 0 degrees. The interpolation of the scalp potential and the derived CSD was based on radial spline-dipoles at a depth of $40 \mathrm{~mm}$. Figure 13a presents the deviations just for the CSD. As before, deviations are smaller with more derise electrode spacing. This result is confirmed by figure 13b. It should, however, be noted that even for unrealistically high spatial sampling a certain error remains which results from the selection of the incorrect (in this case too hard, i.e., spline-dipole at too great a depth) spline functions. As mentioned in the examples above, errors become larger, the more the selected spline dipole depths deviate from the real location of the sources.

Despite all of these shortcomings, it should be noted that within the range of relevant electrode densities (about $8-20 / 2 \pi$; i.e., $25-175$ electrodes) gradients in figure $13 \mathrm{~b}$ exhibit a considerable steepness. This because the gradient can be interpreted as the usefullness to extend the sampling rate.

In a second case, a tangential source was assumed at the distance $\mathrm{r}=40 \mathrm{~mm}$ from the centre of the head. The use of tangential spline functions will produce a result
A
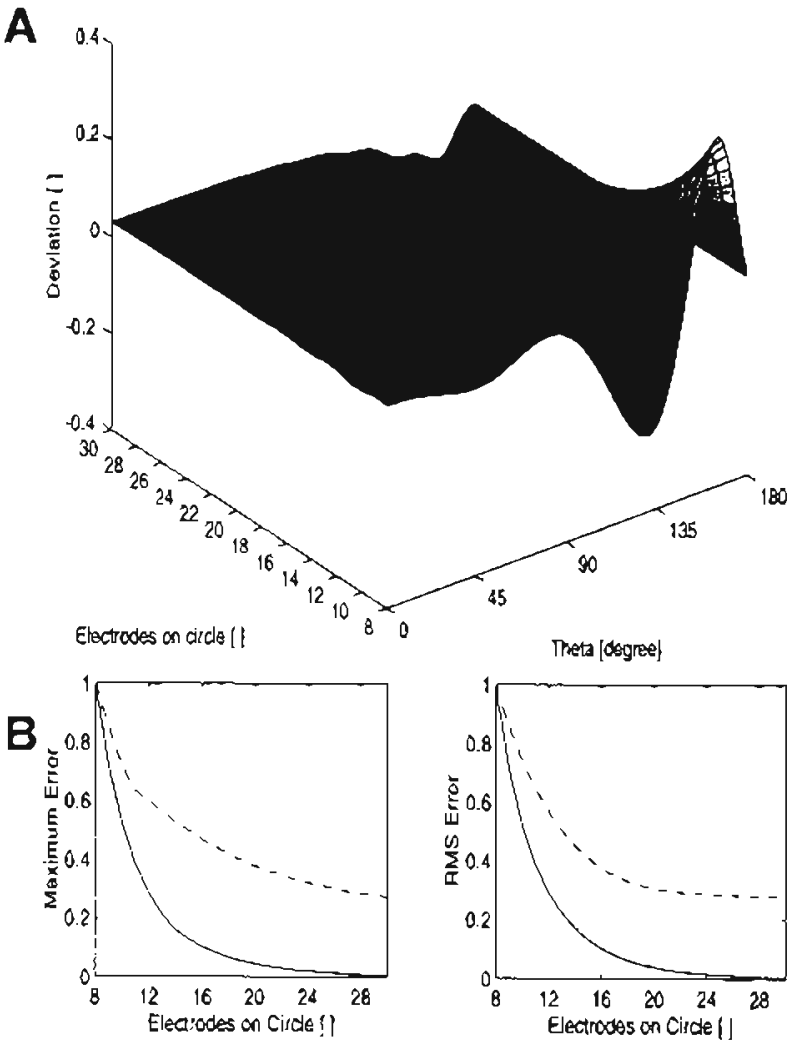

Figure 13. (A) Deviation between the normalized synthetic CSD of a potential generated by a radial dipole at a depth of $20 \mathrm{~mm}$ and the calculated normalized CSD on the base of radial spline-lead-fields at a spline-dipoledepth of $40 \mathrm{~mm}$ in dependency of different electrodespacings between 8 and $30 / 2 \pi$. (B) Between 0 and 1 normalized maximurn and RMS error of the synthetic scalp-potential of a radial source and the spline interpoloted scalp-potential (solid line) both described in figure 130 and the accompanying normalized errors of the CSD (dashed line).

qualitatively similar to the illustrated radial case. To investigate the case further, rndinl leadfield matrices of different depths were used as spline functions. Results are presented in figure 14 in a manner corresponding to the previous figures 10 and 12. All fits are, as expected, significantly worse than for the radial case. We can, however, note one important result: For the selection of extremely stiff spline functions, the fit of tangential leadfields by means of radial spline functions does not become much worse than the choice of splines that correspond to the true depths of the source.

An interpolation can be based on any choice of Green's spline functions. Now we can conclude that the spline-dipole depth should ideally be chosen according to the presumed location of the largest assumed source. Radial spline functions have to be chosen as tangential ones and do not account for the yet to be determined 

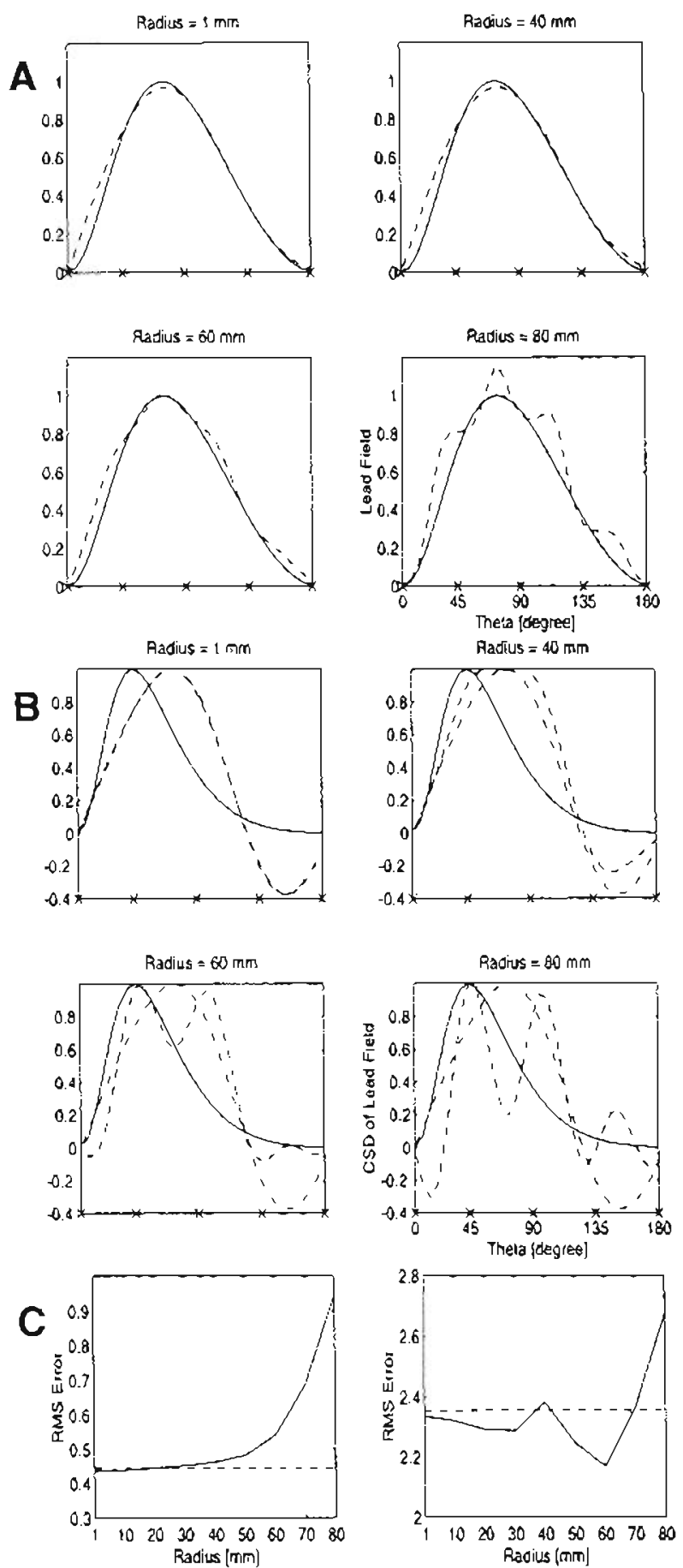

Figure 14. (A) like figure 10 a) but tangential dipole at a depth of $41.5 \mathrm{~mm}$ (radius $=40 \mathrm{~mm}$ ) interpolateo with radial spline-functions with different spline-dipole-rodia. (B) like figure 10b) but tangential dipole at a depth of $41.5 \mathrm{~mm}$. (C) like figure 12 a) but tangential dipole at a depth of 41.5 $\mathrm{mm}$. orientation of the dipole. Extremely 'stiff' spline functions, like that of Perrin and co-workers, allow a good but not optimal fit. When using such 'stiff spline functions, a possible numerical singularity of the leadfield matrices must be excluded.

\section{The Influence of Nolse:}

Next we will consider the impact of noise which has been neglected in all of the computations thus far. Given a certain evoked response, we cannot imagine any way to separate the brain's 'background noise' from an idealised evoked brain signal. Therefore, only technical noise and measurement artifacts can be considered. Technical noise/artifact generally means that the noise at different electrodes is almost independent and therefore spatially not correlated. This is equivalent to a wide spectrum of spatial frequencies. The reader has to distinguish this spatial-from the temporal correlation which in opposite to the spatial one is correlated often and shows a narrower spectrum of time frequencies.

Generally technical noise is much lower than the "background noise" caused by insufficient averaging. Nevertheless, it is not possible to overlook the consequences of technical noise when applying the described deblurring procedures. As high spatial frequencies of the uncorrelated distortions become attenuated by these procedures, artificial ghost sources may appear, just if one electrode value is incorrect. Thus a small movement of just one electrode which produces an incorrect reading may lead to a mistake when interpreting the data.

A simple spatial filter would give less weight to the measured value at one particular electrode, but it can also filter the desired signal. In the high spatial sampling a high electrode number was chosen in order to reach a high spatial resolution and therefor measure the high spatial frequencies. Therefore, only a certain kind of spatial filtering which takes into account knowledge of the spatial frequency characteristics of the brain signal would be appropriate. This kind of filter transforms the signal into the frequency domain where it is multiplied by the spatial frequency characteristic of the transmitting system (head) before it is transformed back into the twodimensional scalp surface. While in the case of simple low-pass filtering, all higher frequencies become attenuated independently of one another, the proposed filter type uses information about the amplitude and phase relationships inherent in the transmitting system, i.e., the volume conductor under consideration.

Using the procedure introduced in this paper, the calculation of the Fourier spectrum of a response to a single impulse is straightforward and an improved filter can be implemented. 
A
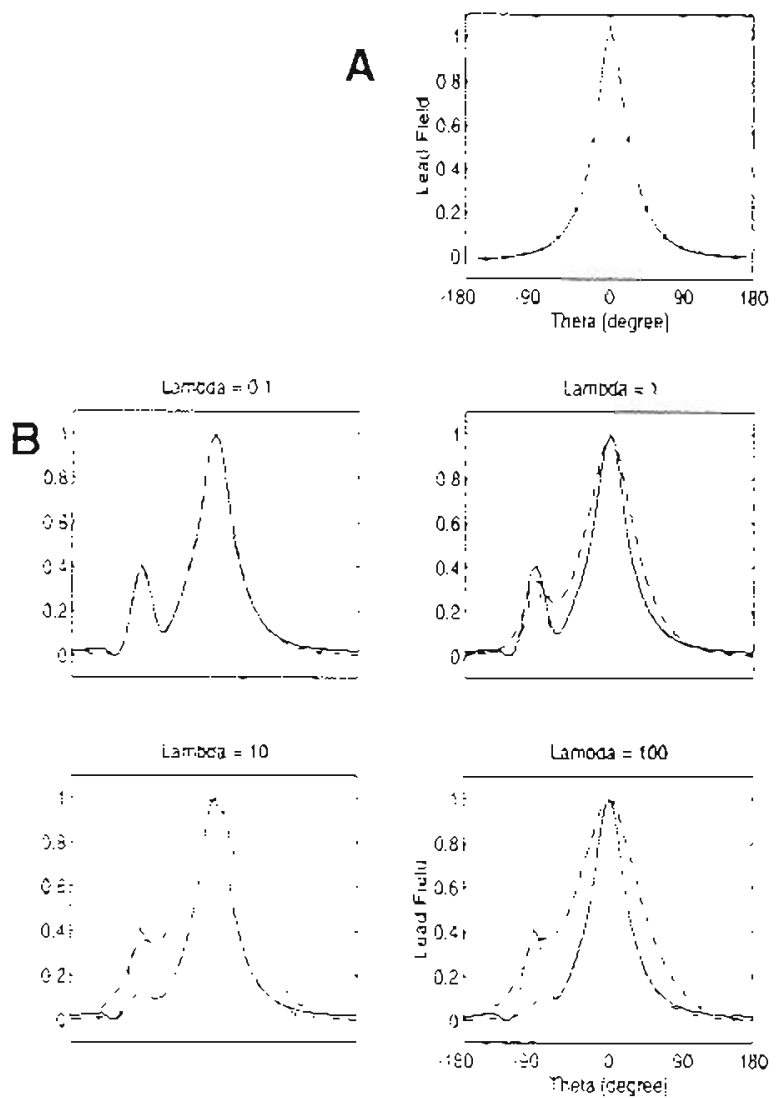

Figure 15. (A) Between 0 and i normolized lead-field of 0 radial synthetic dipole ot a depth of $10 \mathrm{~mm}$. The circles indicates the data used for inverse calculation. The asterisk marks the value which was changed by adding an error of $25 \%$ of the maximum value. (B) To calculate all lines the asterisk in a) in was used instead of the circle at -90 degrees. Solid line: Result of calculation without any noise reduction. Dashed line: Results of calculation with different $\lambda=0.1,1,10$, and 100. All $\beta$; was set to one. Dash dotted line: Results of calculation with different $\lambda$ and on $\beta_{j}$ of 0.25 at -90 degrees. All other $\beta_{j}$ was set to zero.

This procedure will be outlined elsewhere. Here, we will present a simpler form to take noise into account. Instead of the described interpolation, a type of "smoothing" may be used as suggested by Schoenberg 1964 (see also Freeden 1981, Wahba 1981). Formula 4 is extended in the following form:

$$
V_{i}(t)=\sum_{i=1}^{N} p_{i}(t)\left(\lambda \beta_{i}(t) \delta_{i,}+\sum_{n=1}^{\infty} P_{n}\left(\cos \alpha_{i j}\right) \cdot \Gamma^{\text {scalp }(n))}\right.
$$

with: $\delta_{i}= \begin{cases}1, & i=! \\ 0, & i \neq !\end{cases}$

Here $\beta_{i}(t)$ is a positive weight function and $\lambda$ is a positive constant chosen by the user. The effect of these to weightings can be explained easily. With $\delta_{i j}$ these factors act on the diagonal of the inverse gain matrix $G_{i n v}^{s c a l p}$ in which the factors of the "electrode positions" are described with their accompanying fictitious dipoles. Hence, if a special factor $\mathrm{j}$ of the diogonal is weighted stronger in contrast to the other ones this results in a reduction of the strength of the spline dipole $p_{i}$ (inverse calculation) at this position. Therefore the interpolated scalp potential above this special spline dipole is more strong influenced by the surrounding dipoles and the actual value ot the position $;$ looses its weight. Consequently the factors $\beta_{j}$ has to correla te with a weight which describes the reliability of a special factor compared to all the others. The standard-deviation calculated from the baseline could be an example for this procedure. Usually $\beta_{j}$ will be a time independent constant factor for each j but regarding time correlated noise (e.g., noise caused by power lines) $\beta_{j}$ could also be imagined as a known function over time. The constant $\lambda$ gives a measure for the smoothness. The larger the $\lambda$, the weaker the weight of a single value relative to the entity of all measured values. This results in a kind of "smoothing" or approximation respectively.

Figure 15 illustrates this effect: The potential of a radial dipole, located at a depth of $10 \mathrm{~mm}$ and at an angle theta of 0 degrees (solid line in figure 15a) was determined at 16 equidistant positions (o) around a circle. Susequently one of the 16 values (at Theta $=-90$ degrees) was changed by adding an error of $25 \%$ of the maximum value $\left(^{*}\right)$.

In the next step the inverse solution was calculated according to equation 21 using different values of $\lambda$ (title). The corresponding forward calculation produces a potential distribution estimated for 64 locations (figure 15b) dashed line). Increasing $\lambda$ increases the degree of "smoothing". In order to take the simulated artifact into account, an additional $\beta_{j}$ of 0.25 at the position -90 degrees was used while a value of zero was applied for all other positions. The calculated dashed-dotted line determines how the artifact may be accounted for and its effects can be cancelled.

\section{Discussion}

\section{Results for the synthetic data}

Scalp potential data are blurred by the properties of the volume conductor. Therefore, a number of focal activated centers cannot be differentiated from a distributed source and the number of sources cannot be estimated from the scalp potential. By means of the algorithms introduced in the first section of this paper, it can be 
demonstrated that calculations of the CSD, of the cortical map, and in particular of the CSD of the cortical map allow a considerable enhancement of the spatial resolution. These methods, however, require a high spatial sampling, i.e., more electrodes than are included in the standard $10 / 20$ system. If the true source configuration is not known, and this is generally the case, any interpolation may introduce significant errors that may lead to completely wrong interpretations. The only way to avoid this problem is a sufficiently high number of electrodes of 128 channels or greater. The quality of any deblurring method depends on the number of electrodes. An insufficient number will not allow meaningful deblurring. The increment of spatial resolution as well as the error introduced by the interpolation decays exponentially with the number of electrodes. Both functions, however, exhibit gradients that are still steep in the relevant range of 20 to 150 electrodes. Additionally, it must be taken into account, that both of these dependencies must be multiplied with one another in order to estimate the true achievement. Therefore, electrode numbers of up to 300 may be adequate for optimal resolution. Numbers of 64 or more but below 128 may, in certain cases, produce a meaningful CSD (see examples below) but will not allow for a realistic $\mathrm{CSD}$ of the cortical map. In most cases, no reliable conclusions about sources can be drawn from data based on lower electrode numbers such as from a 32-channel system. It also follows that a sufficiently large number of electrodes must be available before averaging across subjects can be attempted. The calculation of grand means may introduce significant errors.

\section{Remarks with respect to MEG:}

Like the electric potential the magnetic field or the gradient of the manetic field is also unambiguously determinable on a closed surface which encloses all sources. Concerning the comparability of MEG experiments realized. with different devices it has recently often been proposed to introduce a basic refering system upon which data of all kinds of systems could be transferred unambiguously. Regarding this demand we have to note again that such a transformation is unique only if the field or the gradient of the field is actually known at every point of the enclosing surface. From what we have said so far we can infer which mistakes are to be expected when transforming MEG data from less than 60 positions on such a basic reference system.

\section{Application to Real Data: The Auditory Evoked N100}

In a further step, the model was tested on EEG data obtained from two subjects, who participated in two sessions each in an auditory oddball task. Two components, N100 and P300, were selected for illustration purposes. The auditory N100 was selected as much of its source configuration is known from both, electrical and magnetic recordings (e.g., Picton et al. 1974, Pantev et al. 1994). In contrast, P300 sources are still under debate and while a general consensus has been reached, that P300 is not likely to result from just one single (e.g., hippocampal) dipolar source per hemisphere, such a view together with a corresponding spatio-temporal modelling has not been completely abandoned.

Methods: Pure tones of 800 and $1200 \mathrm{~Hz}, 50 \mathrm{~ms}$ in duration, $80 \mathrm{~dB}$ SPL, with $5 \mathrm{~ms}$ rise and fall times were presented binaurally through headphones. Stimuli were presented in blocks of 200, with an interstimulus interval of $1.5 \pm 0.2 \mathrm{~s}$. Each block consisted of a pseudorandom sequence containing 20\% of one stimulus ('rare') and $80 \%$ of the other stimulus ('frequent'). The subject was required to respond to one of the two stimuli in a block by pressing a button as fast and as accurately as possible. In each session there were $4(2 \times 2)$ blocks, in which two parameters were counterbalanced: a) response either to rare or to frequent stimuli, and b) the rare stimulus was either the low or the high tone. Each subject participated in 8 sessions, in which the 4 blocks were presented in four different sequences. On odd sessions, the subject used the left hand to press the response key. On even sessions, the right hand was used. The EEG was recorded with zinc electrodes from 64 positions on the head using an Electrocap. Positions included several locations lower on the head such as infraorbital, nasion, outer canthi, FT9/FT10, mastoids, inion and neck. Data were recorded with a 64-channel SYNAMPS (NeuroScan) amplifier system. All 64 channels were referred to $\mathrm{Cz}$. Electrode impedance was below $5 \mathrm{k} \Omega$. Data were amplified at $1 \mathrm{bin} / \mu \mathrm{V}, 16$-bit $A / D$, and sampled at $100 \mathrm{~Hz}$ from $D C-30 \mathrm{~Hz}$. For each task (block), data were recorded continuously. After completion of a session, electrode positions were digitised in three dimensions using a Polhemus digitizer. In addition, the four reference points nasion, inion, left and right preauricular points were digitized.

Artefact-free EEG-epochs were averaged across trials for a 1-s poststimulus interval referred to a 150-ms baseline interval. Scalp potential distribution CSD (Laplacian) and potential distribution on the cortical surface on the basis of the present model were determined at $120 \mathrm{~ms}$ (the maximum of the auditory N100 amplitude) following stimulus onset and for the maximal positivity at $310 \mathrm{~ms}$ (P300).

Results: Figure 17 displays the scalp potential and its CSD distribution for the N100 and for the P300 (see figure 16). The scalp potential distribution suggests a broad distribution of the N100 with a clear fronto-lateral minimum over the right side. The CSD and cortical map are 


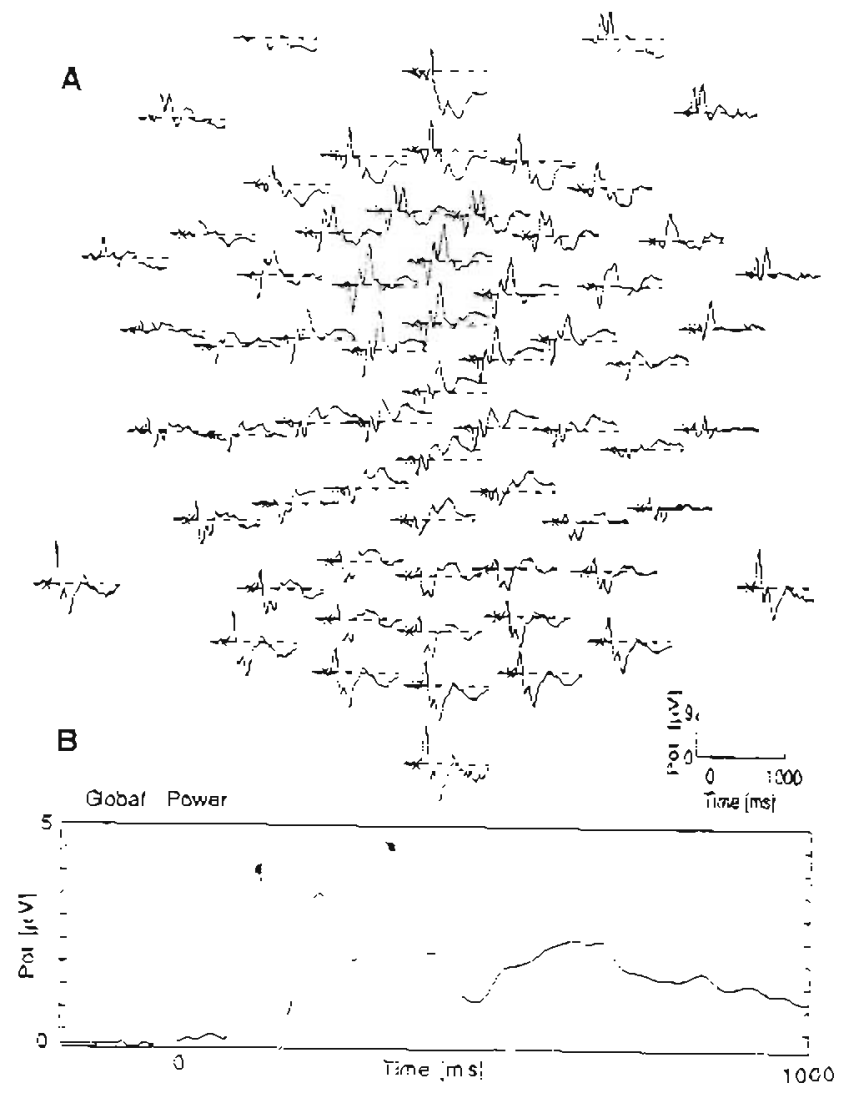

Figure 16. Typical time course a) and global power b) of a 64 channel-data set recorded during the auditory oddball paradigm. The points of time (N100 and P300) illustrated in figure 17 are marked with an asterlsk. very similar and therefore the cortical maps are not shown. From the CSD map two sources become appar ent over each hemisphere, and at least one additional source is suggested by the frontal sink. Qualitatively, this source pattern was apparent for every data set examined.

The simple P300 scalp distribution decays into a multitude of sources and sinks when the CSD is computed. The intricacy of the structure is often smaller than the spatial sampling rate.

Discussion: Based on similar distributions as the one for the N100, but without knowledge of the underlying generator structure, it is common practice to discuss a right frontal involvement in this task. The assumption of just one single source, however, is obviously not true for the N100. The calculation of the CSD and also the cortical map lead to the correct conclusion of two activation centres, visible above both auditory cortices around 120 ms after the stimulus. The calculation of the CSD of the cortical map should produce an even more detailed result. As derived in the previous sections, however, the spatial resolution of 64 electrodes is not sufficient and therefore such a calculation must await measurements with a spatial sampling of 128 channels or more. However, a number of recent studies investigating the auditory N100 and its generators have appeared. Alcaini et al. 1994 and Giard et al. 1994 describe in detail the possible sources of the auditory N100 on the basis of CSD calculated from the grand average across eight subjects. They report "...that even SCD (CSD) mapping dissociates frontal from temporal activities in the N100 scalp distri-
Scalp Potentlal auditory NI 18

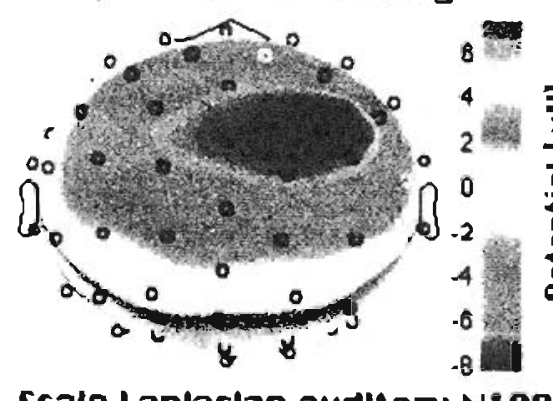

Scalp Laplaclan audit on NiBg

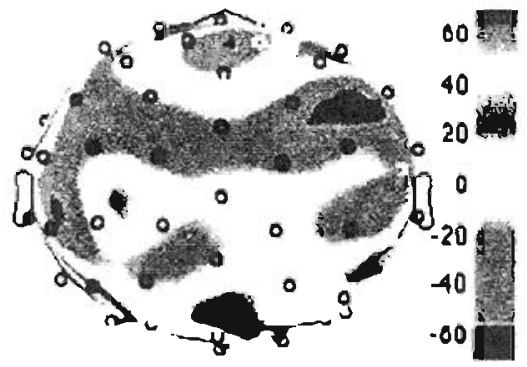

Scalp Potentlal auditory $P 380$

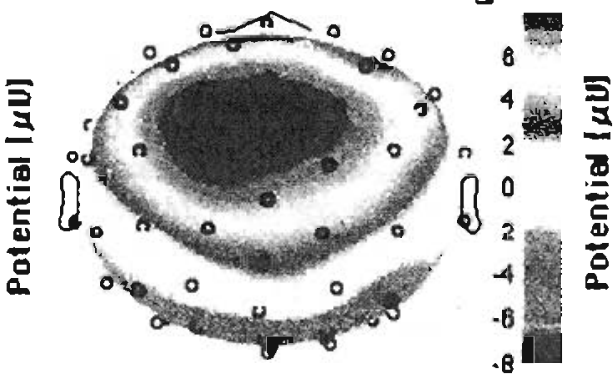

Scajp Laplaclan auditory P3ag

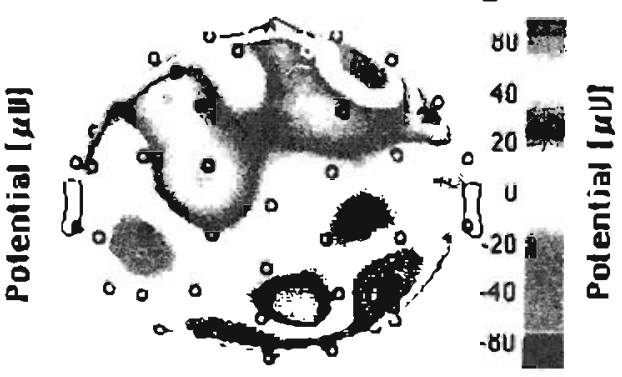

Figure 17. Scalp-interpolation and cortical mapping of the auditory N100 (left) and P300 (right, time courses shown in figure 16) 
bution only if the spatial sampling is sufficently high". Frontal activities could only be detected when 24 electrodes were used, whereas 17 electrodes provided little to no evidence for frontal generators. Of course given the methods dicussed in the present paper, the information from 24 electrodes is still not sufficient. The methods of Giard and coworkers however are not without merit. Averaging over subjects leads in general to spatial lowpass filtering, yet there will be a loss in the spatial resolution of the grand average versus that found in the single subject measurements. Through grand averaging the expected errors due to insufficient spatial sampling will decrease. Based on the findings by Giard et al. it would be interesting and promising to investigate the spatial pattern of activity using more than 100 charunels.

The source configuration for the $\mathrm{P} 300$ seems even more complex and inspection of figure 17 suggests that the present spatial sampling is still too low to arrive at a meaningful identification of the sources. These resuits nevertheless provide further evidence that the P300 cannot be explained by just one single focal activation per hemisphere (e.g., a hippocampal source).

The real data support the view expressed above that nothing meaningful can be inferred about unknown sources from potentials maps derived from 32 electrodes when evenly distributed (e.g., according to the $10 / 20$ system) across the scalp.

\section{References}

Alcaini, M., Giard, M.H., Thevenet, M. and Pernier, J. Dissociation of two frontal components in the N1 wave of the human auditory evoked response Psychophysiol., 1994, 31 : 611-615.

Böhmer K. Spline-Funktionen. B.G. Teubner Stuttgart, 1974.

Braun C., Lutzenberger W., Miltner W., Elbert T. Can subcortical structures generate potentials large in amplitude? In: Brunia, C.H.M., Gaillard, A.W.K., Kok, A., eds. Psychophysiological Brain Research, Vol.1. Tilburg, Tilburg University Press, 1990, pp. 31-35.

Cuffin, B.N. and Cohen, D. Comparison of the magnetoencephalogram and electroencephalogram. Electroenceph. clin. Neurophysiol., 1979, 47:132-146.

Freeden, W. On Spherical Spline Interpolation and Approximation. Math. Meth in the Appl. Sciences, 1981, 3: 551-575.

Gevins, A., Jian, L., Brickett, P., Reutter, B., and Desmond, Y.
Seeing through the skull: advanced EEGs accurately measure cortical activity from the scalp. Brain Topography, 1991, 4: 125-132

Gevins, A., Jian, L., Martin, N.K., Brickett, P., Desmond, J., and Reutter, B. High resolution EEG: 124-channel recording spatial deblurring and MRI integration methods. Electroencph. Clin. Neurophysiol., 1994, 90: 337-358.

Giard, M.H., Perrin, F., Echallier, J.F., Thevenet, M., Froment, J.C. and Pernier, J. Dissociation of temporal and frontal components in the human auditory N1 wave: a scalp current density and dipole model analysis. Electroenceph. clin. Neurophysiol., 1994, 92: 238-252.

Gonzalez, S., Grave de Peralta, R, Lütkenhöner B., Menringhaus, E., and Hoke, M. A strategy for the soultion of the inverse problem using simultaneous EEG and MEG measurements. In Hoke, M., Okada, Y., Erné, S.N., Romani, G.L. (eds.): Advances in Biomagnetism'91: Clinical Aspects. Elsevier, Amsterdam, 1991: 99-100.

Nuñez, P.L., Silberstein, R.B., Cadusch, P.J., and Wijensinghe, R.S. Comparison of high resolution methods having different theoretical bases. Brain Topography, 1993, 5(4): 361364.

Nuñez, P.L., Silberstein, R.B., Cadusch, P.J., Wijensinghe, R.S., Westdorp, A.F., and Srinivasan, R. A theoretical and experimental study of high resolution EEG based on surface Laplacians and cortical imaging. Electroenceph. Clin. Neurophysiol., 1994, 90: 40-57.

Perrin, F., Bertrand, O., and Pernier, J. Scalp current density mapping: value and estemationnfrom potential data. IEEE Transactions on Biomedical Engineering, 1987, 34(4): $283-$ 289.

Perrin, F., Pernier, J., Bertrand, O., and Echallier, J.F. Spherical splines for potential and curre $t$ density mapping. Electroenc. Clin. Neurophys., 1989, 72: 184-187.

Sakatow, P.S. Lehrbuch der höheren Geodäsie. VEB Verlag Technik Berlin, 1957.

Schoenberg, I.J. On trigonornetric spiine interpolation. J. Math. Mech. 13, 1964: 795-825.

Wahba, G. Spline Interpolation and Smoothing on the Sphere, SLAM, J. Sci. Stat. Comput, 1991, 2(1): 5-16.

Yamashita, Y. Theoretical studies on the inverse problem in electrocardiography and the uniqueness of the solution. IEEE Trans. Biomed. Eng. 1982, 29: 719-724.78.

Yamashita, Y. and Takahashi T. Use of the finite element method to determine epicardial from body surface potentials under a realistic torso model. IEEE Trans. Biomed. Eng. 1984, 31: 611-621. 